\title{
Course on integrated optics in the EE undergraduate/graduate curriculum
}

\section{Sheel Aditya}

Sheel Aditya, "Course on integrated optics in the EE undergraduate/graduate curriculum," Proc. SPIE 4588, Seventh International Conference on Education and Training in Optics and Photonics, (28 May 2002); doi: 10.1117/12.468681

SPIE Event: Education and Training in Optics and Photonics 2001, 2001, Singapore, Singapore 


\title{
A course on Integrated Optics in the EE undergraduate/ graduate curriculum
}

\author{
Sheel Aditya ${ }^{1}$ \\ Department of Electrical Engineering \\ IIT Delhi, Hauz Khas, New Delhi - 110016, India
}

\begin{abstract}
During the last decade, there has been an explosive growth in the systems based on optical fibers. These systems require a correspondingly large number of optical components. Most of these components have been realized in integrated-optic form with a number of advantages compared to other forms. Therefore it is extremely important to impart education and training to students in the area of integrated optics. A course on 'Integrated Optics' has been offered during the last few years at the Electrical Engineering Department, Indian Institute of Technology, Delhi (IIT Delhi), India. An integrated optics laboratory in the same department has supported the course. This paper presents various aspects related to this course, viz., the topics covered, laboratory demonstrations, and some of the student projects that have been undertaken over the past few years.
\end{abstract}

Keywords: integrated optics, electrical engineering curriculum, education, photonics

\section{INTRODUCTION}

During the last decade, there has been an explosive growth in the systems based on optical fibers. These systems have applications in communications, radars, sensors, imaging, signal-processing etc. These systems require a correspondingly large number of optical components such as splitters, couplers, multiplexers, switches, filters, modulators etc. Most of these components have been realized in integrated-optic form with a number of advantages compared to other forms, such as those based on optical fibers or micro-optics. Even optical sources, detectors, and optical amplifiers can be realized in integrated-optics. Some of the advantages of integrated-optic components are design flexibility, small size, and low cost.

Integrated-optic devices are now made in the state-of-the-art clean rooms with the same wafer-level processing equipment used in silicon integrated circuit manufacture. The complexity of these devices, as measured by the variety of functions on a chip, the length of devices on a chip, and the number of functional elements, has continued to increase. Fabrication of hundreds of individual passive lightguide circuit devices on a single wafer and over 200 active optical switch elements in a single package module is possible. Actual system applications of integrated devices have been seen in wavelength multiplexed transmission networks, cable TV systems, and high-speed (10 Gbit/s) networks ${ }^{1}$.

It is extremely important to impart education and training to students in the growing and exciting area of integrated optics. A course on 'Integrated Optics' has been offered as an elective during the last few years at the Electrical Engineering Department, Indian Institute of Technology, Delhi (IIT Delhi). The course has been offered at the seniorundergraduate/ graduate level. A small integrated optics laboratory in the same department has supported the course. This paper presents various aspects related to this course, viz., the topics covered, laboratory equipment and lab. demonstrations, and some of the student projects that have been undertaken over the past few years. It should be

1. Current address: Division of Communication Engineering, Blk. S1, School of EEE, Nanyang Technological University, Singapore -639798. Email: esaditya@ntu.edu.sg 
interesting to discuss and compare these aspects with those in the audience who have offered such a course or plan to do so in the future.

\section{DESCRIPTION OF THE COURSE}

\subsection{EE curriculum at IIT Delhi}

The Department of Electrical Engineering at IIT Delhi offers a Bachelor of Technology (B.Tech.) degree in electrical engineering. This is a 4-year programme aiming at a broad-based education in electrical engineering. During the final year of the programme, the students can choose from a number of elective courses to specialize in different aspects of electrical engineering, viz., communications, electronics, computers, control and power. In the same year, the students also undertake a project that runs for two semesters. Each semester extends for 15-16 weeks.

The postgraduate programmes in the EE department lead to M.Tech., M.S.(Research), and Ph.D. degrees. The M.Tech. degree is offered in a number of specializations. These specializations include Communications Engineering and Optoelectronics and Optical Communications. The latter programme is run in collaboration with the Physics Department at IIT Delhi.

The course on Integrated Optics has been offered as an elective course for the M.Tech. students pursuing the specialization in Optoelectronics and Optical Communications (OEOC) or Communications Engineering. The course is also open to the final year undergraduate students in the EE Department. A similar course, offered by the Physics Department, is also available to the M.Tech. (OEOC) students.

\subsection{Topics covered in the Integrated Optics course}

The main objective of the course is to familiarize the students with the fundamental aspects of integrated optics. The covered topics relate to analysis and design of planar, channel, curved, and branching dielectric waveguides; also included are excitation and measurement techniques related to integrated-optic waveguides. After successful completion of the course, the students are able to undertake design of simple channel waveguides; they are also able to plan and carry out some of the important measurements on planar and channel waveguides. Usually there is no time in the lectures to cover specific materials, different waveguide configurations, or functional devices; however, these aspects inevitably enter the picture during laboratory demonstrations and student-projects.

As a pre-requisite, the students should have gone through a basic course in electromagnetics so that they have familiarity with concepts of wave propagation in infinite media, and reflection/transmission at boundaries between media.

The topics covered in the course are listed below. Also mentioned is the typical number of lectures for each topic. Books and/or reference material found useful for different topics are also indicated.

- Introduction ${ }^{2,3}: 1$ lecture

Brief history of optical communication; advantages of integrated optics configuration; typical integrated-optic components; examples of capabilities

- Review of wave propagation ${ }^{2}: 3$ lectures

Wave vector, properties of plane waves

Reflection/transmission at a dielectric interface, phase matching, total internal reflection, reflection and transmission coefficients 
- Planar slab guides ${ }^{2,3,4}: 8$ lectures

Ray optics, b-V curves for TE/TM modes, number of modes, Goos-Hänchen shift, Fields-based analysis, characteristic equation for TE mode, graphical solution, $\omega-\mathrm{k}_{\mathrm{z}}$ diagram Asymmetric slab guide, characteristic equation, dispersion relation Power flow, symmetric and asymmetric guides, effective guide thickness Radiation modes, characteristic equation, graphical solution

- Planar graded-index waveguides ${ }^{2,3}: 3$ lectures

WKB method, normalized characteristic equation, parabolic index profile, ray trajectory

- Channel waveguides: 8 lectures

Effective-index method ${ }^{2}$; Marcatilli's method ${ }^{3,5}$; Gaussian approximation ${ }^{6}$; design of single mode guide ${ }^{3}$

- Bent and curved waveguides: 5 lectures

Corner bend waveguides; S-bends ${ }^{3}$

Curved waveguides ${ }^{2}$, plane-wave spectrum of finite width beams, attenuation constant for curved waveguides ${ }^{2,5}$

Equivalent index profile ${ }^{6}$, modal field offset

- Branching waveguides ${ }^{3}: 3$ lectures

Tapered waveguides, branching waveguides, super-modes, scattering loss in a Y-junction; various types of power dividers

- Waveguide excitation ${ }^{3,5}: 4$ lectures

End coupling; prism coupling, m-lines; grating coupling

- Measurements and evaluation ${ }^{7}: 3$ lectures

Measurement of propagation constant, mode profile

Measurement of waveguide parameters, step-index and graded-index planar waveguides

Measurement of transmission loss

\section{INTEGRATED OPTICS LABORATORY}

\subsection{Equipment and facilities}

A laboratory has been developed to carry out work related to integrated optics. The laboratory has proved very helpful for instruction purposes. The laboratory contains facilities for fabrication of glass ion exchange planar and channel waveguides, and equipment to support evaluation of planar and channel waveguides. Initially, silver ion-exchange waveguides were fabricated using soda-lime glass. Later on, potassium ion-exchange waveguides using BK-7 glass were fabricated. E-beam mask for channel waveguides and waveguide circuits were made at ITI, Bangalore. Metalization and photolithography etc., required for channel waveguides, were carried out in the microelectronics laboratory of CARE, IIT Delhi. 
The laboratory contains the following equipment/facilities. Details of equipment are provided in the appendix.

- Ion-exchange furnace

- Optical measurement set-up for channel waveguides

- Stereo-zoom microscope

- Imaging/Viewing facilities

- Prism coupling set-up for planar waveguides

- Lapping and polishing

- Fiber preparation facilities

\subsection{Demonstrations and experiments}

The students get an opportunity to design, fabricate and test integrated-optic waveguides. The students carry out the following demonstrations and experiments:

- Design of waveguides

Analysis and design of waveguides using software developed at IIT Delhi. The software is based on Beam Propagation Method.

- Fabrication of planar and channel glass ion-exchange waveguides

$\mathrm{Ag}^{+}$and $\mathrm{K}^{+}$ion-exchange planar waveguides; $\mathrm{K}^{+}$ion-exchange channel waveguides; surface and buried channel waveguides; edge polishing

- Planar waveguides

Prism coupling, m-lines, number of modes, propagation constants, reconstruction of index profile; measurement of loss

- Channel waveguides

Fiber cleaving, laser-to-fiber coupling, fiber-to-waveguide coupling, measurement of loss

Viewing and capturing mode profile

- Waveguide circuits

Testing of S-bends and Y-junction power splitters

\section{STUDENT PROJECTS}

The following is a list of some of the projects undertaken by bachelor's and master's students. Contrary to the popularity of software projects with the students, most of these projects involved fabrication and painstaking testing.

- B.Tech. students:

- Design, fabrication and testing of ion-exchanged integrated -optic guides

- Investigation of fiber compatibility of ion-exchanged integrated-optic channel guides

- Fabrication of fiber array blocks for pig tailing of integrated-optic waveguides 
- $\quad$ M.Tech. students:

- Optimization of ion-exchange process for single mode fiber compatible integrated optic waveguide

- Computer aided design and technology development of integrated optic waveguides

- Analysis of integrated optical power divider

- Path design by minimization of bending losses for integrated -optic waveguides

- Determination of process parameters for two step K-Na ion exchange for integrated optic waveguides

- Investigations on S-bends in integrated-optic waveguides

- Development of pig tailing schemes for integrated-optic waveguides

- Loss measurements in integrated-optic waveguides

O Investigation of S-bends for integrated-optic power splitters and pig tailing for integrated-optic waveguides

\section{CONCLUSION}

A course on Integrated Optics has been described. The course is targeted at master's students but is open to final year bachelor's students as well. In the lectures, analysis of planar and channel waveguides is emphasized. The course is supported by a laboratory in which students can design, fabricate and test integrated-optic waveguides. This gives the students an opportunity to understand first hand the precision and patience required for work in this area. In addition, students undertake projects that reinforce, extend, and complement the topics covered in the lectures.

\section{ACKNOWLEDGEMENT}

Planning for the course and development of the laboratory were done jointly with Prof. D. Chadha. She shared teaching in the course once and also shared supervision of many student projects. The laboratory was funded under a project from Govt. of India. Support of Professors A. K. Ghatak and Sudhir Kumar, and help of Bharat Gupta, Sunita Kothari, Mahendra and J. Krishnaswamy are also gratefully acknowledged. Contribution of students, whose projects are mentioned here, is also thankfully acknowledged.

\section{APPENDIX}

- Optical measurement set-up for channel waveguides

Optical bench

Mechanical and electrical translation stages

Optical sources and power meter (1310 and $1550 \mathrm{~nm})$

- Stereo-zoom microscope

Reflection and transmission

Long travel stage

Camera attachment

- Imaging/Viewing facilities

CCD camera for visible light and monitor

Infrared camera $(400-1900 \mathrm{~nm})$

Image-acquisition card and image-processing software 
- $\quad$ Prism coupling set-up for planar waveguides

Rotary and translation stages

High-index prisms and prism holders

$\mathrm{He}-\mathrm{Ne}$ laser tube $(630 \mathrm{~nm})$

- Lapping and polishing

Lapping and polishing machine with variable speed and timer

Jig for edge polishing

Lapping and polishing compounds

- Fiber preparation facilities

Fiber cleaver

Fiber ribbonizer

Hot-jacket stripper

Ribbon fiber fan-out cords

\section{REFERENCES}

1. Edmond J Murphy (Ed.), 'Integrated Optical Circuits and Components, ' Marcel Drekker Inc., N.Y., 1999.

2. Donald L. Lee, 'Electromagnetic Principles of Integrated Optics,' John Wiley \& Sons, N.Y., 1986.

3. H. Nishihara, M. Haruna, and T. Suhara, 'Optical Integrated Circuits, ' McGraw-Hill Book Co., N.Y., 1989.

4. T. Tamir, 'Integrated Optics', Springer Verlag, 1975.

5. D. Marcuse, 'Light Transmission Optics', Van Nostrand Reinhold, N.Y., 1973.

6. François Ladouceur and John D. Love, 'Silica-based buried channel waveguides and devices', Chapman \& Hall, N.Y., 1996.

7. S. I. Najafi (Ed.), 'Introduction to Glass Integrated Optics,' Artech House, Boston, 1992. 